
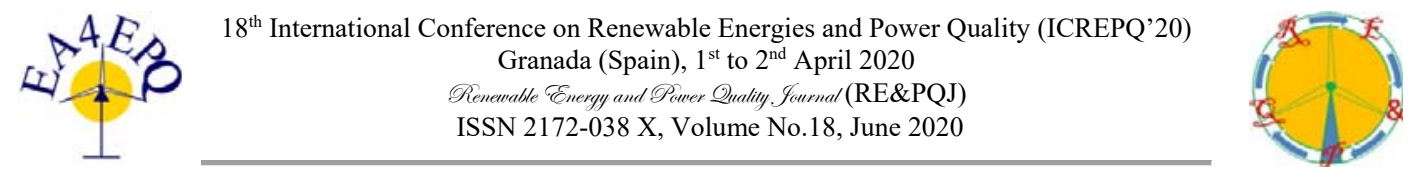

\title{
A Site Characterization Index for Continuous Power-Quality Monitoring based on Higher-Order Statistics
}

\author{
Olivia Florencias-Oliveros ${ }^{1}$, Jose-María Sierra-Fernández, Juan-José González-de-la-Rosa, \\ Agustín Agüera-Pérez, Manuel-Jesús Espinosa-Gavira, José-Carlos Palomares-Salas \\ ${ }^{1}$ Research Group PAIDI-TIC-168: Computational Instrumentation and Industrial \\ Electronics (ICEI). University of Cádiz. Area of Electronics. Higher Polytechnic School \\ of Algeciras. \\ Av. Ramón Puyol S/N. E-11202-Algeciras-Cádiz-SpainPhone/Fax number: +0034 986812685 \\ olivia.florencias@uca.es
}

\begin{abstract}
The current high penetration rate of distributed energy resources in the smart grid has set up a challenge for energy management and consequently for the supply monitoring and assessment of power quality. Currently, new types of electrical disturbances have appeared, usually multiple, due to the uncontrolled connection of non-linear loads that damage sensitive electronic equipment. For this reason, continuous monitoring is the most effective way to characterize the power system behaviour in order to be able to improve determining the origin of the fault and to have weight bearing to claim damages.

Likewise, more holistic indicators that would compute the different network states and deal with big data management in terms of compression and scalability of the measurements are needed. In this context, this article presents a statistical characterization in order to monitor in continuous the voltage supply in a consumer network node through a voltage quality index based on higher-order statistics. The results allow us to conclude that higher-order statistics are capable of establishing a continuous, reliable performance that characterizes the deviations of the voltage supply waveform in an average consumer installation. The method could lead to more feasible solutions for power quality surveillance in the smart grid.
\end{abstract}

Key words. Power Quality (PQ), Power system metering, Continuous monitoring, Data compression; Higher-Order Statistics (HOS)

\section{Introduction}

The high penetration of renewable energy resources as expected in the future Smart Grid (SG) is a challenging scenario for energy management and consequently it is driving the design of Power Quality (PQ) monitoring equipment. The SG demands more efficient systems and monitoring algorithms that, deployed in a new measurement infrastructure, would create a more flexible power system [1]. Indeed, new PQ disturbances are expected to impact the network due to the uncontrolled connection of non-linear loads at different levels in the electrical network, from LV to HV. Thus, the most efficient monitoring strategy for different multiple disturbances is a fundamental issue in the power system modernization. For this reason, several areas of knowledge are converging in the study of PQ (e.g., Statistical Signal Processing, Instrumentation and Measurements for Internet of Things, IoT). New analytical tools should be developed so that to comply with a more complex PQ analysis in real time and online monitoring [2],[3],[4],[5].

So far, in Europe the measurement campaigns have been based on widely accepted meters and developed in accordance with the norm IEC 61000-4-30 [6]. However, more and more implementations of new measurement solutions are more adapted to the requirements of the specific campaign to be developed. PQ monitoring demands a more efficient data management strategy which ensures flexible reporting (e.g., time-varying scales), improving the monitoring of different sites and allowing measurement traceability and repeatability [7],[8]. Moreover, nowadays grid performance analysis conveys the characterization of multiple measurement locations, which results in the production of huge amounts of data that require organization (e.g. the problem of Big Data). This in turn must be based on the elimination of redundant and erroneous information, and on the formulation of new indicators that bring together the greatest possible significance of the measurements, adapted to the customers requirements [9],[10].

Along with the inherent deregulation of the energy market that comes with the renewable energy introduction, PQ measurements are called to contribute to improvements in compatibility between consumers and grid operator solutions. More flexible and ad hoc measurements are needed with the aim of managing the massive data generated during a measurement campaign that provide redundant information. Throughout this argument, the idea of using more understandable indicators lies not only for energy suppliers but also for the end users thereof; producers and consumers 
(prosumers) since the norm EN 50160 does not discriminate responsibilities between suppliers and endconsumers in the Point of Common Coupling (PCC) [10], [11].

Also, the standard (EN50160) describes the supply of voltage and deviations until the PCC (the physical connection between the grid and the end-user) Fig.1. From the consumer side, the user must fulfil the harmonized EMC Standards (Article 6 of the EMC Directive) accomplishing the immunity and emission parameters of the user. In the SG context, two-flow energy will take place at the PCC. Despite that, current standards have been developed when linear (resistive) consumers dominate the power distribution networks, and they do not match for supply and consumption and not covering the endconsumer real behaviour. The impact of non-linear devices over voltage is not characterized, neither the introduction of more distributed resources from an increasingly flexible network [12]. As a result, the power system measurements do not reflect the real load behaviour. There is a need of characterization through real-measurements from endconsumers that reflects the interaction between the loads (current) and the voltage.

With all the above, and according to the concept of a PQ index, it should provide response to different concerns related to the two main types electrical disturbances: continuous and discrete. These two strategies are usually based on techniques that compress the acquired time-series cycle-by-cycle and normalize the signals extracting their useful information in the time and frequency domains. For instance, the site indices may unify measurements and compute individual weekly percentiles at different physical levels along the network. Nevertheless, far from being updated, PQ norms and standards do not still gather sufficiently flexible measurement strategies that should be included in future networks and implemented in new meters. According to this idea, if permanent monitoring is desired, it is necessary to incorporate new measurement indexes and ensure that their compliance remains within $95 \%$ confidence interval within a week [6]. In other words, current power measurements are definitely insufficient to achieve a full characterization of the current electrical disturbances and consequently to bound its causes within the network scheme in the SG context [13].

In relation to the feature extraction stage, its importance lies in the previous preprocessing that guarantees the maximum probability of detecting not only the events when performing permanent monitoring but also the network behaviour under normal operating conditions. All that information is crucial in order to be implemented in the future power recognition systems [14], [15], [16].

The PQ campaigns must produce reliable data that accomplish the metrological requirements with a Bayesian perspective reducing the uncertainty in the process control, continuously measuring the characteristics of the network under test [17].

Many publications have put the focus on characterizing individual PQ events. Not many researchers have improved the real-time events detection and characterization [18]. Neither, many examples of characterization of multiple events in real-time monitoring can be founded [19]. The development of PQ technology lacks a generalized approach to the problem that can handle all different events, that is, single and multiple events [18],[19],[20].

The present proposal helps characterize the network behaviour and its deviation from the ideal steady state through an index based on Higher-Order Statistics (HOS). The performance of the method is studied in order to be introduced in S Class instruments according the IEC 61000-4-30 [6] and adding flexibility to measurements and their reporting that match the SG context.

The main goals of the research are described hereinafter. First, HOS are capable of characterizing the waveform distortion based on monitoring their Probability Density Function (PDF), reporting parameters such as symmetry, amplitude and tail deviations of the waveform PDF. Thus, for continuous analysis, this work proposes a global PQ deviation index based on HOS. Secondly, the work postulates PQ estimators based on HOS evolution that help characterize the waveform in a consumer installation under normal operating conditions at different measurement scenarios in a permanent regime (daily/weekly). This contributes to improve future prediction tools based on artificial intelligence. In third and final place, a real-time solution is developed using $\mathrm{LabVIEW}^{\mathrm{TM}} 116$.

The paper is structured in five sections. The first one introduces the theoretical background related to the PQ indices and the fundamental definitions of the HOS. The second part, exposes the measurement and analysis framework. The analysis results are made in the third one. This is followed by, the discussion of the results and the contribution of the proposal which is made in different scenarios. Finally, in the conclusions, authors recommend how the method can be used in order to perform different types of analysis in field.

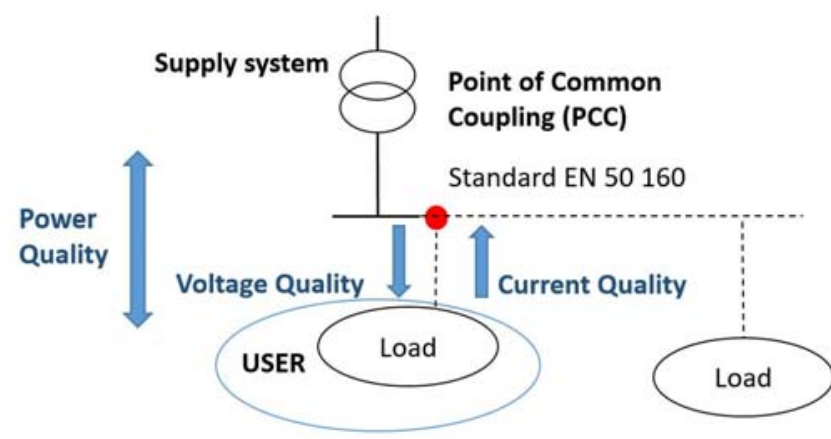

Fig. 1 The EN50160 rules voltage until the PCC. Non-linear loads deteriorate current waveforms in short period of time. The distorted current waveforms (non-sinusoidal) increase voltage deviation. As Ohm's law, the voltage waveform is an impedance function of the user network and the current waveform. A method that characterizes the voltage waveform behaviour the combined effect of those events that have an origin in the grid and those derived from the user's behaviour are detected from the voltage waveform monitoring using HOS statistics. 


\section{Theoretical background}

\section{A. $P Q$ indices}

New measurement solutions propose managing the information from energy meters by adopting different strategies, converting big data from each smart meter into a series of probability distributions. Calculating the pair wise distance between load proles; and transform each long series of demand data into a single two-dimensional (2D) scatter plot [16].

In the $\mathrm{SG}$ scenario, PQ must be traceable and more understandable for both, customers and energy suppliers. In this sense, the main contribution of this proposal consists of a bi-dimensional space of higher-order statistics (2D HOS plane) that characterizes the bimodal distribution of a voltage wave form, compressing the time-domain information, and computing the evolution of the quality vector of the waveform shape cycle-to-cycle, Fig. 3. The present proposal contributes to solve some PQ monitoring issues: data compression, a scalable index and a continuous monitoring based on a more feasible solution that uses the HOS computation. The proposal differs from the conventional PQ measurements which are based on the second-order (e.g., the power spectrum and the Total Harmonic Distortion, THD). The method is considered as an additional solution that contributes to improve the PQ report with a global indicator that informs about the waveform shape status.

The method helps characterize the point under test and monitor the network holistic, allowing to trigger a more specific offline analysis in case the network parameters fall outside normal operating conditions, not fulfilling the contractual requirements.

\section{B. The HOS-based PQ deviation index}

Different HOS estimates have been proposed through the last decade to infer new statistical characteristics associated with data from a non-Gaussian time-series in a predominant Gaussian background floor, which can be theoretically considered as a result of the summation of different noise processes.

Within the PQ disturbance detection context, the targeted electrical disturbance is always considered non-Gaussian, while the floor is assumed to be a stationary Gaussian signal [21]. Thus, using HOS would help detect the perturbation, e.g. qualitatively, with a rough approximation, but allowing detection within a frame of a continuous measurement campaign. The main mathematical formulations regarding HOS have been previously introduced in [21].

Based on the premise that, HOS have shown success and application in other fields of Science and Technology (e.g., vibration mechanics, acoustic detection of insects such as termites [22]), they are suitable for the new power grid scenario. HOS are good for non-linear system characterization and less sensitive to noise than other signal processing methods.
As it has been advanced before, this tool contributes significantly to the studies of classification of electrical disturbances since it addresses not only the instantaneous power parameters of the signal, but also those associated with its shape [21], [23]. This circumstance confers higher-order statistics suitable characteristics to analyse the new types of multiple disturbances that occur in the electrical network which are mainly due to the introduction of renewable resources.

The strategy consists of calculating three statistics: the variance, the skewness and the kurtosis of the signal under test. Each record analysed results in this triplet, which in the case of an ideal supply voltage signal takes the values $(0.5,0.0,1.5$, respectively). The proposed index will attempt to quantify the differences between an ongoing measurement and the former ideal triplet. As can be seen, the index introduces shape parameters of the signal that are not traditionally included in the norms and standards [13], which usually deal with second-order parameters such as Vrms or THD. Likewise, by gathering the measurements of each record in three statistical parameters, the memory savings are notable, which allows us to conclude that an index of these characteristics is suitable for dealing with large amounts of data.

This triplet $(0.5,0.0,1.5)$, is assumed as the steady state values from which to measure deviations in the HOS planes. The PQ deviation index is a function of the summation of the specific deviations of each individual statistic (variance, skewness, and kurtosis) with respect their nominal values on the HOS planes. The index have previously formuled in [24], and is given by the generic expression in Eq. (1):

$\Delta \mathrm{t}$ : measurement interval.

sij : $j$-th statistic associated with the $\mathrm{i}-$ th period.

$\hat{s} j$ : nominal $\mathrm{j}$-th statistic.

$\mathrm{M}$ : number of periods contained in $\Delta \mathrm{t}$.

$P Q_{\Delta t}=\frac{\sum_{i=1}^{M}\left|v a r_{i}-v \hat{a} r\right|+\left|s k_{i}-\hat{s k}\right|+\left|k u r_{i}-k \hat{u} r\right|}{M}$,

where var; sk and kur represents the variance, skewness and kurtosis respectively.

They HOS definitions related to sag and swell detection were previously defined in [21], an extended definition in the HOS plane analysis when sag, swell, harmonics and transients taken place were made in [24], to detect fundamental frequency deviations using HOS [25].

The contribution of the paper is improved measurement state performance and capabilities in PQ instruments under a real-time analysis campaign proposing a set of parameters (higher-order statistics) that extract new signal features more than traditional PQ analysis, and combined enhance significantly PQ indication through a deviation index, and 2D PQ patterns. This is special relevant within the instrumentation field in a Smart Grid scenario of continuous monitoring. 


\section{Measurement and analysis framework}

All the measurements have been made in the installations at the University of Cádiz in Andalusia (Spain) during a six-week campaign. The goal was monitoring the $50-\mathrm{Hz}$ voltage waveform in $\mathrm{LV}$ at a sampling rate of $25 \mathrm{kHz}(500$ $\mathrm{Sa} /$ cycle).

The devices used in the acquisition system are the chassis NI cDAQ-9188 of National Instruments Technology, using an analogue input module NI-9225 serie C. The chassis was connected via Ethernet to a PC with LabVIEW $^{\text {TM }}$ which developed continuous analysis.

The algorithm calculates the three statistics making use of a sliding window, with no overlapping, cycle-by-cycle. In a subsequent stage, the values are subtracted to the ideal quantities, their absolute values calculates and the added to finally result in the PQ index in Eq. (1).

In order to stablish a procedure to study the different patterns, all the measurements were analysed offline a posteriori in MATLAB ${ }^{\mathrm{TM}}$. The method follows the next steps:

1. Extracting HOS and computing PQ cycle-to-cycle in LabVIEW TM. This is considered as a first time compression when calculating the triplet of statistics for each cycle.

2. Store the HOS and the PQ time-series in a weekly trend data-set.

3. Normalizing and pre-processing the HOS and the PQ week time series in MATLAB ${ }^{\mathrm{TM}}$ using the histograms weights. In order to depict a representative percentage of the HOS and the PQ time-series, 1 in 1000 data per bin, were selected in order to extract the PQ trend with the minimum of information. Using this technique, authors have reduced the data set memory storage.

4. Plot the cumulative distribution function (CDF) and the histograms of the HOS and the PQ indicator.

5. Selection of the HOS and the PQ range based on weekly trend measurements.

6. Plot the PQ time-series. Represent the HOS day pattern feature in different colour maps along the weeks.

7. Extracting day colour map matrices for PQ pattern detection.

\section{Analysis results}

Fig.4, shows different histograms of the variables involved in the method: the PQ index, the variance, the skewness, and the kurtosis. All the measurements have been obtained during six weeks in the same connection point under test. The first three figures represent the cumulative density function estimate (CDF) of each index, the PQ and the individual HOS. Down, in Fig.2, the probability density functions (PDF) are also represented for each indicator. In the PDF, each $\mathrm{N}$ values is equal to the relative number of observations in the bin such that sum $(\mathrm{N})$ is 1 .

According to the weekly histograms results, while the skewness and the kurtosis seem to have a most stable range while the variance shows a wider one. The results obtained, mainly those related to variance helps understand that the majority of fluctuations that occur during a week are associated with changes in variance (amplitude changes). In addition, changes in the tails present a probability of occurrence evidenced by the deviation observed in the kurtosis index. This fact contrasts with the less frequent changes in the symmetry of the distribution, a question which is de facto assimilated as a working hypothesis for future experiments in the same voltage supply point. However, we must bear in mind that skewness is another term of the PQ index, which will charge specific weight in certain measuring points that experience deviations in the symmetry of the signal.

Next, and in order to obtain daily patterns of 2D graphs, one week evolution is shown based on the variance versus kurtosis graphs in Fig. 3. The values with the maximum temporal resolution of $0.02 \mathrm{~s}$, cycle by cycle, have been considered. For each day of the considered week, its intensity graph of variance versus kurtosis (2D colour maps of HOS) has been considered, being the skewness obviated for the reasons stated above. Similarities have been found between patterns of different days within a week. The measurements took place from Monday to Sunday, from November 13th to 28th, 2017. During these two weeks all the cycles of the measured signals were processed except a lack of $7.2 \%$ of week 1 data and a $0.6 \%$ of week 2 data (missing 12 hours and 1-hour monitoring data respectively) according to a loose of connection between the acquisition unit and the pc during the monitoring campaign.

The CDF and PDF analysis help stablish a characterization analysis of the wave-shape in the point under test. For a weekly/ monthly campaign, the most representative indices seem to be variance and kurtosis. Nevertheless, it is important to say that based on author's experience, skewness can be useful in strategies more focused on small length windows and event detection. The main contribution to the histograms' analysis is to set a more realistic measurements to the individual ranges of indices and detect their region of maximum probability within the whole campaign.

The time domain analysis helps detect the effect of the waveform deviation computed by the statistics. Also, allows to identify the individual contribution of each indicator to the global PQ. Indeed, in the network under test, the PQ day fluctuates depending on the day of the week, the hourly trend of the network, the energy usage during working or non-working hours. During the night, in general the PQ cycle-to-cycle can fluctuate between 0.01-0.02 428 values. During the morning the deviation of the PQ increases reaching maximum 0.04 values during the noon. Also, between 13:00-16:00h there is a drop in the PQ because this is the lunch schedule in Spain. A second increase of the index occurs in the evening schedule, since the university is open until 22:00h. Finally, during midnight the PQ decreases again, recovering the minimum values. Indeed, 2D graphics allow visualizing such behaviour by emphasizing the areas of signal persistence throughout the hours, days and weeks. 

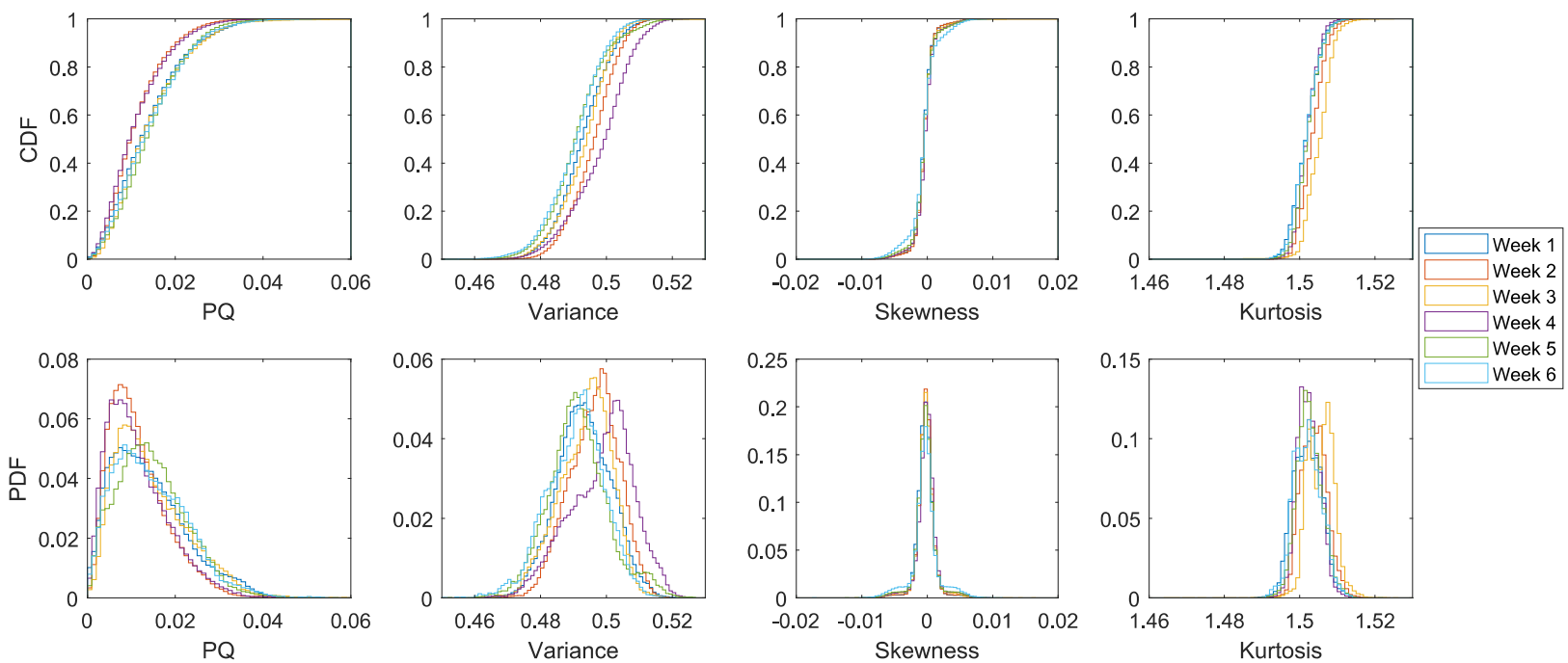

Fig. 2. Histograms of the different weekly indices based on the cumulative density function (CDF) and the probability density function (PDF).

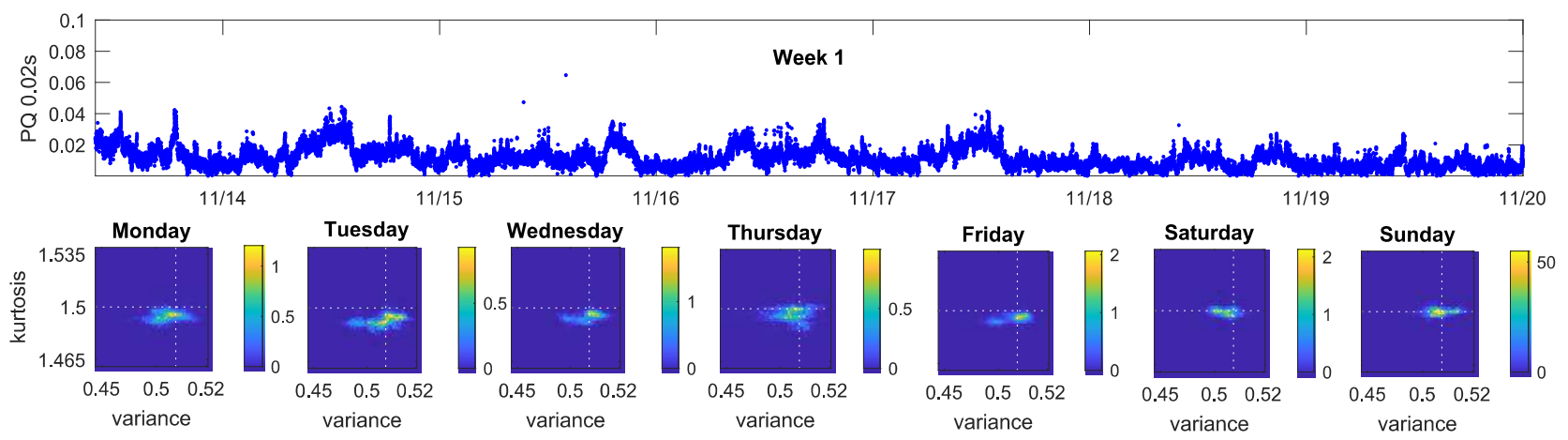

Fig. 3. Representation of the $\mathrm{PQ}$ index time-series along the first week of the monitoring campaign and their trend, and different $2 \mathrm{D}$ colour maps of day-to-day patterns of HOS.

\section{Conclusions}

Related to practical use of the proposed PQ procedure based on HOS and the PQ index pattern models:

The method assumes that loads and customer deviations are intrinsic characteristics of the network not enough quantified by the PQ conventional measurement analysis. HOS have demonstrated to provide information regarding the waveform shape in terms that the rest of the existing indicators do not.

Characterize the waveform distribution under normal operating conditions, in continuous, helps conduct PQ analysis closer to reality. This is relevant when in previous publications the limits from which a PQ problem can be detected through HOS (amplitude, fundamental frequency, symmetry, wave shape) have been previously defined.

The model of PQ relative to a node is based on real-time measurements. The model and graphs are very useful when analysing PQ impact on the node under test under normal operating conditions and for subsequent analysis.

The procedure of analysis improved PQ reporting, and visualization of the network state. In this proposal, data compression techniques related to continuous analysis incorporate intelligent data management from feature extraction and compression in time (different interval reports) and in space (2D graphs) more intuitive for both, specialist in the field and consumers. This can be useful in site characterization and in future performance analysis of networks in the SG.

The PQ pattern and in the HOS space (clusters regions) can provide thresholds more aligned to the instantaneous state of the network, based on the clients' footprint, taking into account time, and the waveform characteristics, as well as to determine the percentage of the data more convenient to store according to the PQ monitoring objectives in the point under test.

Finally, the procedure of analysis is important, in order to accomplish the monitoring strategies and objectives in the advanced-monitoring infrastructure such as smart meters with PQ functionalities.

\section{Acknowledgement}

This work is supported by the Ministry of Economy, Industry and Competitiveness (Spanish Government) and the EU (AEI/FEDER/UE) via the project TEC201677632-C3-3-R - COntrol andManagement of Isolable NanoGrids: Smart Instruments for Solar forecasting and 
Energy Monitoring. Also, our gratitude goes to the Andalusian Government for supporting the Research Group PAIDI-TIC-168, in Computational Instrumentation and Industrial Electronics (ICEI).

\section{References}

[1] M. Bollen, S. Bahramirad. Is there a place for power quality in the smart grid? IEEE (Ed.), 16th International Conference on Harmonics and Quality of Power (ICHQP), 2014, pp. 713-717.

[2] M. H. Bollen, I. Gu. Signal Processing of Power Quality Disturbances. Wiley-IEEE Press, 2006.

[3] D. S. P. M. de Paulo F. Ribeiro, Carlos Augusto Duque. Power Systems Signal Processing for Smart Grids. Wiley - IEEE, 2013.

[4] O. N. Gerek, D. G. Ece. Compression of power quality event data using $2 \mathrm{~d}$ representation. Electric Power Systems Research 78 (Issue 6) (2008) 1047-1052.

[5] C.-I. Chen, Y.-C. Chen, Y.-C. Chin, C.-H. Chen. Integrated power quality monitoring mechanism for microgrid, IEEE Transactions on Smart Grid, 9 (6) (2018) 6877-6885.

[6] IEC, IEC 61000-4-30. Electromagnetic compatibility (EMC), part 4: Testing and measurement techniques. Section 30: Power quality measurement methods.

[7] M. Bollen, P. Baumann, Y. Beyer, R. Castel, J. Esteves, S. Fajas, W. Friedl, S. Larzeni, J. Trhulj, F. Villa, L. Stro m. Guidelines for good practice on voltage quality monitoring, in: IEEE (Ed.), 22nd IEEE International Conference on Electricity Distribution (CIRED), Vol. 1, 2013, pp. 1-4.

[8] R. R. Mohassel, A. Fung, F. Mohammadi, K. Raahemifar. A survey on advanced metering infrastructure, International Journal of Electrical Power \& Energy Systems 63 (2014) 473-484.

[9] Design aspects for large PQ monitoring systems in future smart grids, IEEE Power and Energy Society General Meeting, IEEE, 2011.

[10] J. Meyer, M. Klatt, P. Schegner. Power quality challenges in future distribution networks, in: IEEE (Ed.), 2nd IEEE PES International Conference and Exhibition on Innovative Smart Grid Technologies, Vol. 1, 2011, pp. 1-6.

[11] D. D. Ferreira, J. M. de Seixas, A. S. Cerqueira, C. A. Duque, M. H. Bollen, P. F. Ribeiro. A new power quality deviation index based on principal curves. Electric Power Systems Research 125 (2015) 8-14.

[12] A. Moreno-Munoz, J. de-la Rosa, M. Lopez-Rodriguez, J. Flores-Arias, F. Bellido-Outerino, M. R. de Adana. Improvement of power quality using distributed generation. International Journal of Electrical Power \& Energy Systems 32 (10) (2010) 1069-1076.

[13] J. V. Milanovic, M. H. J. Bollen, N. Cukalevski. Guidelines for monitoring power quality in contemporary and future power networks - results from CIGRE/CIRED JWG C4.112, in: C4-115 2014, 2014

[14] R. Duda, P. Hart, D. Stork. Pattern Classification, 2nd Edition, Wiley-Interscience, 2000.

[15] J. S. Pattern Recognition-Concepts, Methods and Applications, Springer, 2001.

[16] R. J. Hyndman, X. A. Liu, P. Pinson. Visualizing big energy data: Solutions for this crucial component of data analysis, IEEE Power and Energy Magazine 16 (3) (2018) 18-25.

[17] J.-M. Pou, L. Leblond. ISO / IEC guide 98-4: A copernican revolution for metrology, IEEE Instrumentation \& Measurement Magazine 21 (5) (2018) 6-10.

[18] S. Khokhar, A. A. B. M. Zin, A. S. B. Mokhtar, M. Pesaran. A comprehensive overview on signal processing and artificial intelligence techniques applications in classification of power quality disturbances. Renewable and Sustainable Energy Reviews 51 (2015) 1650-1663.
[19] M. 621 K. Saini, R. Kapoor. Classification of power quality events: a review. International Journal of Electrical Power \& Energy Systems 43 (1) (2012).

[20] O. P. Mahela, A. G. Shaik, N. Gupta. A critical review of detection and classification of power quality events. Renewable and Sustainable Energy Reviews 41 (2015).

[21] A. Agüera-Pérez, J.-C. Palomares-Salas, J.-J. G. de-la Rosa, J.-M. Sierra-Fernández, D. Ayora-Sedeño, A. MorenoMuñoz. Characterization of electrical sags and swells using higher-order statistical estimators. Measurement (Ed. Elsevier) 44 (Issue 8) (2011) 1453-1460.

[22] J. J. G. de la Rosa, I. Lloret, C. Puntonet, R. Piotrkowski, A. Moreno. Higher-order spectra measurement techniques of termite emissions. a characterization framework. Measurement 41 (1) (2008) 105-118.

[23] J.-J. G. de-la Rosa, A. Agüera-Pérez, J.-C. PalomaresSalas, J.-M. Sierra-Fernández, A. Moreno-Muñoz. A novel virtual instrument for power quality surveillance based in higher-order statistics and case-based reasoning. Measurement (Ed. Elsevier) 45 (Issue 7) (2012) 1824-1835.

[24] O. Florencias-Oliveros, J.-J. González-de-la-Rosa, A. Agüera-Pérez, and J.-C. Palomares-Salas, "Power quality event dynamics characterization via $2 \mathrm{D}$ trajectories using deviations of higher-order statistics," Meas. J. Int. Meas. Confed., vol. $125,2018$.

[25] Olivia Florencias-Oliveros, Agustín Agüera-Pérez, JoséMaría Sierra-Fernández, Juan-José González-De-La-Rosa, JoséCarlos Palomares-Salas, Manuel-Jesús Espinosa-Gavira. IEEE 9th International Workshop on Applied Measurements for Power Systems (AMPS), 2018. 